\title{
The PASTEL catalogue: 2016 version ${ }^{\star}$
}

\author{
Caroline Soubiran, Jean-François Le Campion, Nathalie Brouillet, and Laurent Chemin
}

\author{
Laboratoire d'Astrophysique de Bordeaux, Univ. Bordeaux, CNRS, UMR 5804, 33615 Pessac, France \\ e-mail: caroline.soubiran@u-bordeaux.fr
}

Received 11 March 2016 / Accepted 4 May 2016

\begin{abstract}
The bibliographical compilation of stellar atmospheric parameters $\left(T_{\mathrm{eff}}, \log g,[\mathrm{Fe} / \mathrm{H}]\right)$ relying on high-resolution, high signal-tonoise spectroscopy started in the eighties with the so-called $[\mathrm{Fe} / \mathrm{H}]$ catalogue, and was continued in 2010 with the PASTEL catalogue, which also includes determinations of $T_{\text {eff }}$ alone, based on various methods. Here we present an update of the PASTEL catalogue. The main journals and the CDS database have been surveyed to find relevant publications presenting new determinations of atmospheric parameters. As of February 2016, PASTEL includes 64082 determinations of either $T_{\text {eff }}$ or $\left(T_{\text {eff }}, \log g\right.$, [Fe/H]) for 31401 stars, corresponding to 1142 bibliographical references. Some 11197 stars have a determination of the three parameters $\left(T_{\mathrm{eff}}, \log g,[\mathrm{Fe} / \mathrm{H}]\right)$ with a high-quality spectroscopic metallicity.
\end{abstract}

Key words. catalogs - stars: abundances - stars: atmospheres - stars: fundamental parameters

\section{Introduction}

The knowledge of stellar atmospheric parameters $\left(T_{\text {eff }}, \log g\right.$, $[\mathrm{Fe} / \mathrm{H}])$ is crucial in many reasearch areas related to the physics of stars and galaxies. In particular the atmospheric parameters are needed before the chemical composition of a star can be estimated.

PASTEL is a bibliographical catalogue compiling determinations of stellar atmospheric parameters (Soubiran et al. 2010). It provides $\left(T_{\text {eff }}, \log g,[\mathrm{Fe} / \mathrm{H}]\right)$ determinations obtained from detailed analyses of high-resolution $(R \geq 25000)$, high signal-tonoise spectra $(S / N \geq 50)$, carried out with the help of model atmospheres. PASTEL is the continuation of the effort made by Giusa Cayrel de Strobel to make high-quality iron abundances available to a wide community in a convenient way. Six editions of the $[\mathrm{Fe} / \mathrm{H}]$ catalogue have been published (Cayrel de Strobel et al. 1980, 1981, 1985, 1992, 1997, 2001). PASTEL follows the same philosophy with one modification: it also provides effective temperatures $T_{\text {eff }}$ determined from various methods. We decided to include $T_{\text {eff }}$ because it is a critical parameter in spectroscopic analyses. Knowing $T_{\text {eff }}$ precisely leads to lower uncertainties in abundances (see e.g. Jofré et al. 2014, 2015). It is thus helpful to gather studies providing precise determinations of $T_{\text {eff }}$, even if not based on high-resolution spectroscopy. In particular, the increase in the accuracy of angular diameter measurements of stars by interferometry has provided a number of direct measurements of $T_{\text {eff }}$ through the fundamental relation, very important because largely model-independent. We found it relevant to include them in PASTEL.

PASTEL is available through VizieR at CDS. It is a popular catalogue, queried about 15000 times per month on average. The initial version (Soubiran et al. 2010) included 30151 records. Two updates have been provided, in 2011 (31724 records) and 2013 (52045 records). We present in this paper the new

\footnotetext{
$\star$ The PASTEL catalogue is available in electronic form at the CDS (http://vizier.u-strasbg.fr/viz-bin/VizieR?-source=B/ pastel).
}

version of PASTEL, as of Febuary 2016, which now has 64082 records. Section 2 describes the contents of the revised release of PASTEL in terms of stellar parameters. Section 3 presents its stellar content.

\section{Description of the catalogue}

\subsection{Star names, coordinates, and magnitudes}

Only stars with known equatorial coordinates in Simbad are listed in PASTEL. Owing to this restriction, several studies could not be included, especially in the regions of stellar clusters where the stars are often ambiguously identified. This restriction, however, ensures that PASTEL can be cross-matched to other catalogues.

The Simbad database has recently been cleaned and some stars that were in previous versions of PASTEL have been removed because of their unknown or too uncertain coordinates. Fifty-two stars not identified in Simbad can be found in the 2MASS catalogue (Cutri et al. 2003) and so the 2MASS coordinates have been adopted.

Like in the previous versions of PASTEL, some spectroscopic binaries remain problematic because their parameters could be determined separately from high spectral resolution spectroscopy, while only one entry is available for both components in Simbad. These stars are not included in PASTEL.

For each star recorded in PASTEL, Simbad was queried to retrieve its equatorial coordinates (ICRS, epoch J2000) and its $B, V, J, H, K$ magnitudes. The catalogue can thus be searched by position on the sky and magnitude interval. There are 31401 different stars in the current version of PASTEL.

\subsection{Effective temperature $T_{\text {eff }}$}

The $63797 T_{\text {eff }}$ determinations in PASTEL span a wide range from $\sim 2300 \mathrm{~K}$ to $10^{5} \mathrm{~K}$ with a median value of $6000 \mathrm{~K}$. 

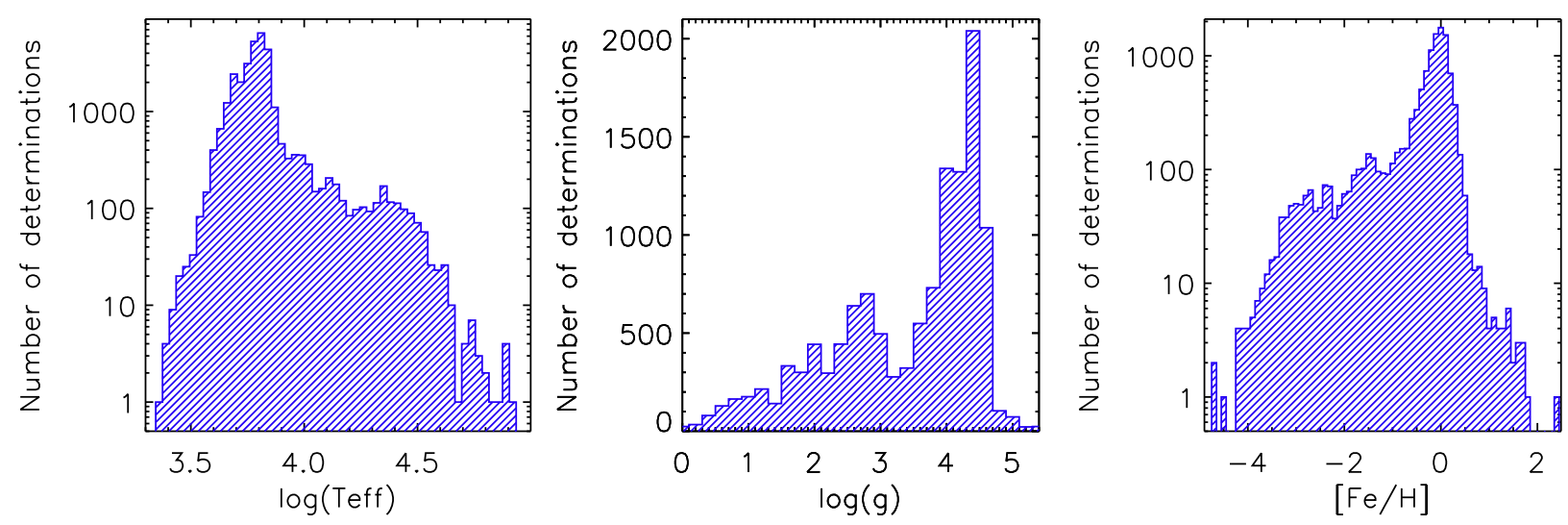

Fig. 1. Histogram of effective temperatures, surface gravities, and metallicities in PASTEL.

The histogram is shown in Fig. 1. The vast majority of $T_{\text {eff }}$ determinations correspond to FGK stars.

If quoted in the articles, uncertainties on $T_{\text {eff }}$ are provided. They vary from less than $5 \mathrm{~K}$ for some FGK stars (e.g. Kovtyukh et al. 2006; Ramírez et al. 2014) to $\sim 5000 \mathrm{~K}$ for some OB stars (e.g. Sokolov 1995; Latour et al. 2013). Translated into relative errors, their median value is $1.1 \%$. It is worth noting that there is a variety of definitions or procedures to evaluate $T_{\text {eff }}$ uncertainties that are not homogeneous. When a star has multiple $T_{\text {eff }}$ values, the standard deviation shows how these different determinations agree with one other, and reflects both internal and external errors, including differences in temperature scales. Nearly half of the stars in PASTEL have at least two $T_{\text {eff }}$ values from which the standard deviation can be computed. Its median value is $0.76 \%$, demonstrating that the authors agree quite well in general, even if they have not used the same observing material and methods. There are outliers, however; some stars have $T_{\text {eff }}$ determinations in significant disagreement (83 and 343 stars have a standard deviation larger than $10 \%$ and $5 \%$, respectively). To discard discrepant determinations, it is safer to adopt a median instead of an arithmetic mean if an averaged $T_{\text {eff }}$ value is needed.

A new feature in the 2016 version of PASTEL is to include several recent references providing effective temperatures from angular diameters and total flux at Earth for 151 stars (261 determinations by Boyajian et al. 2012a,b, 2013, 2015; von Braun et al. 2014; Heiter et al. 2015). They provide fundamental values of $T_{\text {eff }}$, which define the absolute temperature scale. Figure 2 displays the comparison of these fundamental values to the photometric or spectroscopic $T_{\text {eff }}$ listed in PASTEL. The fundamental $T_{\text {eff }}$ are cooler by $51 \mathrm{~K}$ (median) than the spectroscopic or photometric values, with a median absolute deviation (MAD) of $96 \mathrm{~K}$. For most stars, the $T_{\text {eff }}$ differences span at least $200-300 \mathrm{~K}$. We have investigated the most extreme differences, i.e. those larger than $500 \mathrm{~K}$. They mainly come from less reliable determinations in the earliest bibliographic references, or from the most uncertain determinations in González Hernández \& Bonifacio (2009) who sometimes quote errors of several hundred K. Other large differences are difficult to explain without going into the details of the publications.

\subsection{Logarithm of surface gravity, log g}

Surface gravities $\log g$ are usually derived by the method of ionization equilibrium or by the parallax method. A novelty of the 2016 version of PASTEL is to have $\log g$ determinations based

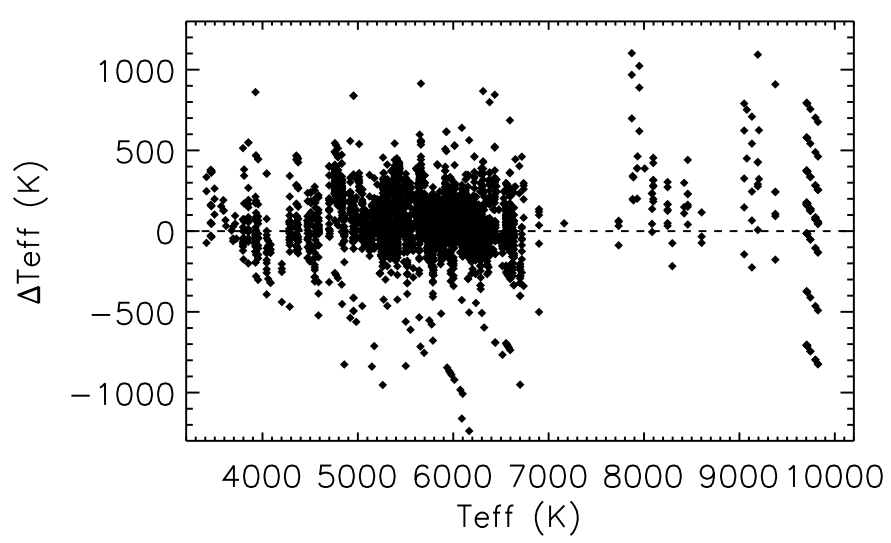

Fig. 2. Difference between fundamental effective temperatures and other estimates listed in PASTEL $\left(\Delta T_{\text {eff }}=T_{\text {other }}-T_{\text {Teff }}\right)$ for 151 stars. The fundamental $T_{\text {eff }}$ ( $x$-axis) are based on direct measurements of angular diameters and total flux at Earth. We note that several stars have up to six determinations of fundamental $T_{\text {eff }}$ listed in PASTEL.

on asteroseismology. Several studies combining asteroseismology and spectroscopy are included (e.g. Creevey et al. 2012; Takeda \& Tajitsu 2015).

The distribution of the $27178 \log g$ determinations is shown in Fig. 1. A clear separation at $\log g \simeq 3.2$ between the giants and the dwarfs and subgiants can be seen. Dwarfs and subgiants represent $\sim 65 \%$ of the $\log g$ determinations.

When available in the papers, errors on $\log g$ are provided in PASTEL. They have a median value of $0.10 \mathrm{dex}$, which agrees nicely with the median standard deviation (0.09 dex) for stars having at least two $\log g$ determinations available.

\subsection{Metallicity, $[\mathrm{Fe} / \mathrm{H}]$}

Only $[\mathrm{Fe} / \mathrm{H}]$ determinations based on high-resolution, high signal-to-noise spectra are recorded in PASTEL. High spectral resolution was considered to start at $R=25000$, and high $\mathrm{S} / \mathrm{N}$ at 50 . In a few cases, determinations that do not exactly fulfil the $\mathrm{S} / \mathrm{N}$ requirements have been included. For instance, the highresolution spectroscopic study of extremely metal-poor stars by Aoki et al. (2013) has been entirely included even if a fraction of the spectra have a $\mathrm{S} / \mathrm{N}$ lower than our limit owing to the very high interest of the targets.

The distribution of the $26718[\mathrm{Fe} / \mathrm{H}]$ determinations is shown in Fig. 1. The $[\mathrm{Fe} / \mathrm{H}]$ determinations range from -4.80 
Table 1. Median atmospheric parameters of the 36 most studied stars in PASTEL.

\begin{tabular}{|c|c|c|c|c|c|c|}
\hline HD & $T_{\text {eff }}$ & $N_{T_{\text {eff }}}$ & $\log g$ & $N_{\log g}$ & {$[\mathrm{Fe} / \mathrm{H}]$} & $N_{[\mathrm{Fe} / \mathrm{H}]}$ \\
\hline HD 006582 & $5308 \pm 44$ & 37 & $4.50 \pm 0.10$ & 27 & $-0.83 \pm 0.07$ & 28 \\
\hline HD 009826 & $6155 \pm 51$ & 39 & $4.14 \pm 0.08$ & 28 & $+0.09 \pm 0.04$ & 29 \\
\hline HD 010700 & $5331 \pm 41$ & 45 & $4.50 \pm 0.10$ & 32 & $-0.52 \pm 0.04$ & 35 \\
\hline HD 019445 & $5985 \pm 74$ & 48 & $4.39 \pm 0.11$ & 40 & $-1.96 \pm 0.11$ & 43 \\
\hline HD 019994 & $6150 \pm 59$ & 34 & $4.15 \pm 0.10$ & 29 & $+0.21 \pm 0.04$ & 29 \\
\hline HD 022049 & $5084 \pm 51$ & 37 & $4.54 \pm 0.08$ & 30 & $-0.09 \pm 0.05$ & 29 \\
\hline HD 022879 & $5857 \pm 59$ & 43 & $4.29 \pm 0.06$ & 34 & $-0.84 \pm 0.02$ & 35 \\
\hline HD 023249 & $5023 \pm 77$ & 30 & $3.80 \pm 0.04$ & 25 & $+0.11 \pm 0.06$ & 28 \\
\hline HD 027371 & $4990 \pm 50$ & 25 & $2.70 \pm 0.12$ & 23 & $+0.11 \pm 0.06$ & 25 \\
\hline HD 028305 & $4915 \pm 69$ & 27 & $2.70 \pm 0.13$ & 24 & $+0.15 \pm 0.05$ & 25 \\
\hline HD 029139 & $3891 \pm 52$ & 28 & $1.20 \pm 0.26$ & 26 & $-0.16 \pm 0.16$ & 26 \\
\hline HD 034411 & $5860 \pm 30$ & 35 & $4.22 \pm 0.04$ & 22 & $+0.06 \pm 0.05$ & 25 \\
\hline HD 039587 & $5947 \pm 27$ & 30 & $4.47 \pm 0.04$ & 22 & $-0.04 \pm 0.03$ & 25 \\
\hline HD 061421 & $6582 \pm 68$ & 53 & $4.00 \pm 0.02$ & 36 & $-0.01 \pm 0.03$ & 43 \\
\hline HD 063077 & $5723 \pm 70$ & 28 & $4.10 \pm 0.11$ & 24 & $-0.86 \pm 0.08$ & 27 \\
\hline HD 064090 & $5400 \pm 38$ & 34 & $4.50 \pm 0.20$ & 27 & $-1.69 \pm 0.13$ & 27 \\
\hline HD 075732 & $5252 \pm 56$ & 31 & $4.40 \pm 0.09$ & 24 & $+0.34 \pm 0.06$ & 25 \\
\hline HD 076932 & $5860 \pm 40$ & 37 & $4.10 \pm 0.10$ & 30 & $-0.90 \pm 0.04$ & 32 \\
\hline HD 084937 & $6290 \pm 68$ & 40 & $4.00 \pm 0.06$ & 33 & $-2.11 \pm 0.11$ & 33 \\
\hline HD 094028 & $5995 \pm 61$ & 38 & $4.19 \pm 0.13$ & 29 & $-1.44 \pm 0.07$ & 30 \\
\hline HD 095128 & $5887 \pm 25$ & 34 & $4.30 \pm 0.04$ & 30 & $+0.01 \pm 0.02$ & 30 \\
\hline HD 102870 & $6103 \pm 43$ & 43 & $4.14 \pm 0.06$ & 29 & $+0.14 \pm 0.04$ & 31 \\
\hline HD 103095 & $5054 \pm 64$ & 53 & $4.65 \pm 0.12$ & 42 & $-1.35 \pm 0.06$ & 43 \\
\hline HD 106516 & $6208 \pm 61$ & 36 & $4.40 \pm 0.10$ & 26 & $-0.73 \pm 0.05$ & 28 \\
\hline HD 109358 & $5887 \pm 43$ & 35 & $4.42 \pm 0.07$ & 23 & $-0.21 \pm 0.02$ & 25 \\
\hline HD 114710 & $6000 \pm 50$ & 38 & $4.43 \pm 0.06$ & 22 & $+0.06 \pm 0.04$ & 28 \\
\hline HD 114762 & $5871 \pm 63$ & 41 & $4.17 \pm 0.07$ & 32 & $-0.72 \pm 0.05$ & 33 \\
\hline HD 122563 & $4583 \pm 17$ & 46 & $1.20 \pm 0.20$ & 39 & $-2.65 \pm 0.09$ & 42 \\
\hline HD 124897 & $4300 \pm 45$ & 41 & $1.60 \pm 0.12$ & 37 & $-0.54 \pm 0.06$ & 38 \\
\hline HD 140283 & $5690 \pm 65$ & 63 & $3.58 \pm 0.13$ & 54 & $-2.48 \pm 0.13$ & 57 \\
\hline HD 146233 & $5799 \pm 24$ & 33 & $4.45 \pm 0.03$ & 27 & $+0.03 \pm 0.02$ & 26 \\
\hline HD 193901 & $5745 \pm 82$ & 31 & $4.46 \pm 0.12$ & 26 & $-1.08 \pm 0.09$ & 26 \\
\hline HD 194598 & $5962 \pm 62$ & 37 & $4.27 \pm 0.06$ & 31 & $-1.12 \pm 0.06$ & 32 \\
\hline HD 201891 & $5900 \pm 47$ & 38 & $4.31 \pm 0.09$ & 32 & $-1.05 \pm 0.05$ & 32 \\
\hline HD 217014 & $5775 \pm 25$ & 34 & $4.33 \pm 0.08$ & 26 & $+0.20 \pm 0.01$ & 26 \\
\hline HD 224930 & $5362 \pm 87$ & 26 & $4.40 \pm 0.18$ & 20 & $-0.76 \pm 0.12$ & 25 \\
\hline
\end{tabular}

Notes. The error is the median absolute deviation (MAD) obtained from the $N$ determinations available in the catalogue.

to +2.40 . The solar metallicity is by far the most frequent value. More than $70 \%$ of the $[\mathrm{Fe} / \mathrm{H}]$ determinations are between -0.50 and +0.50 .

Errors on $[\mathrm{Fe} / \mathrm{H}]$ are also provided when available and have a median value of 0.06 dex. For the 5061 stars that have at least two $[\mathrm{Fe} / \mathrm{H}]$ determinations, the median standard deviation is 0.05 dex. The median standard deviation is even lower ( 0.04 dex) for FGK stars $\left(4000 \leq T_{\text {eff }} \leq 6500 \mathrm{~K}\right)$ more metal-rich than $[\mathrm{Fe} / \mathrm{H}]=-1.0$ dex. These good values of dispersion suggest that the errors quoted in the publications are in general not underestimated. For metal-poor FGK stars the median standard deviation is 0.09 dex. A few stars (79) exhibit large discrepancies in $[\mathrm{Fe} / \mathrm{H}]$ determinations, with a standard deviation larger than 0.3 dex. These are mainly metal-poor stars and A-, B-, and M-type stars.

A flag in PASTEL indicates when the metallicity determination relies on all metals (as often is the case for $\mathrm{M}$ dwarfs), or when $[\mathrm{Fe} / \mathrm{H}]$ is corrected for non-local thermodynamic equilibrium effects, or when $[\mathrm{Fe} / \mathrm{H}]$ has been measured from FeII lines.

The methods of spectral analysis have evolved; they are more automated and are applied to large numbers of spectra.

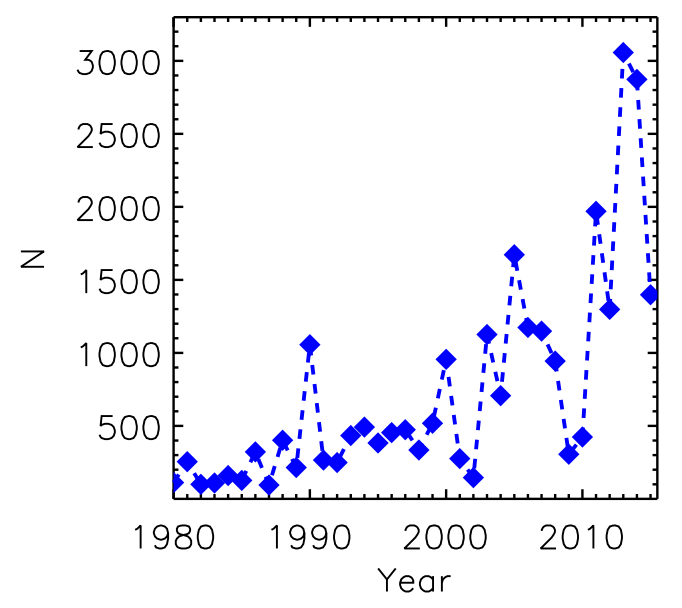

Fig. 3. Number of $[\mathrm{Fe} / \mathrm{H}]$ determinations per year of publication.

Consequently, the number of $[\mathrm{Fe} / \mathrm{H}]$ determinations is rapidly increasing, as is shown in Fig. 3, which displays the number 


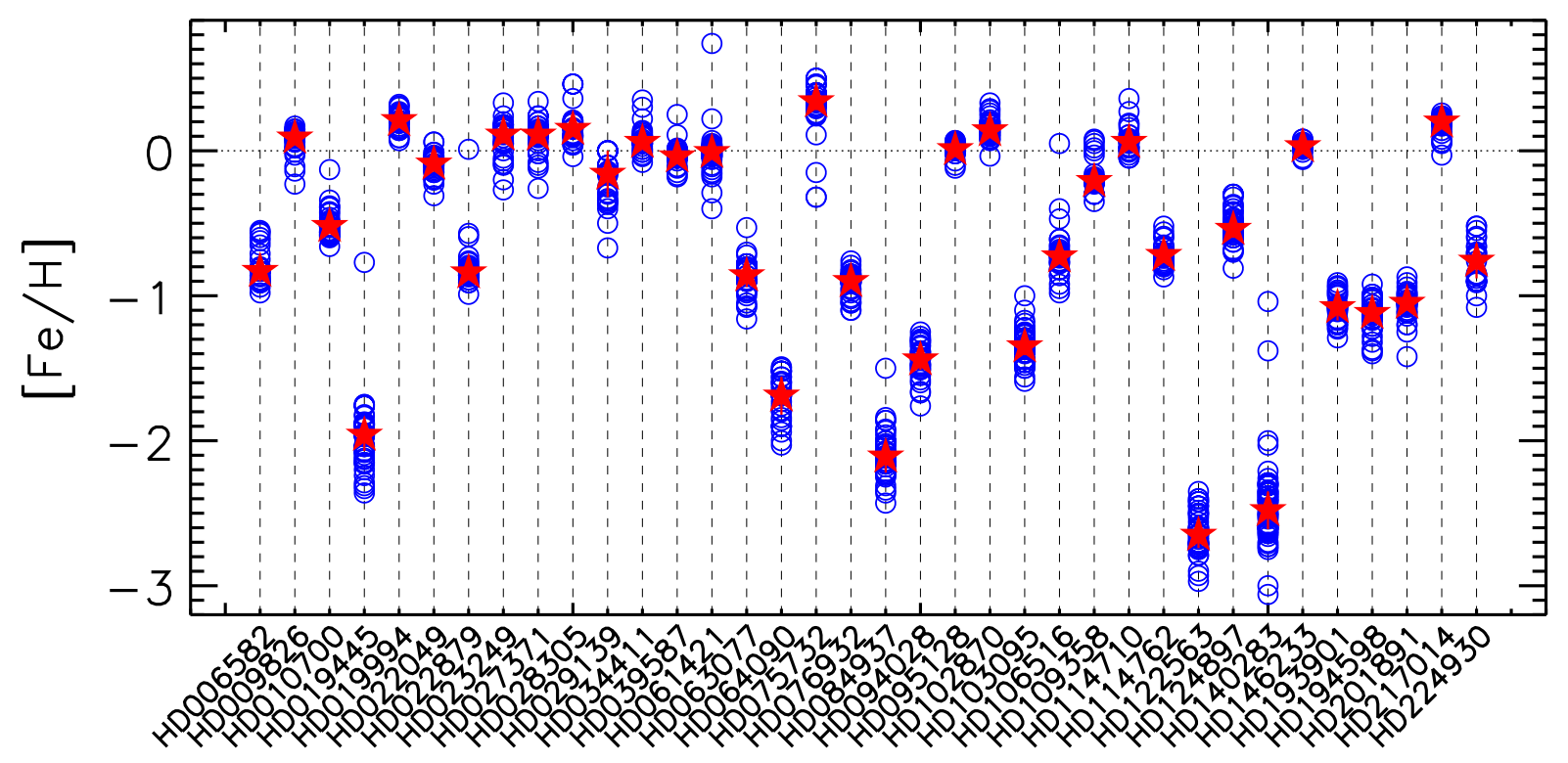

Fig. 4. Metallicities reported in PASTEL for the 36 most studied stars listed in Table 1 (open circles). The median value is represented by a red star.

of $[\mathrm{Fe} / \mathrm{H}]$ determinations per year of publication. The increase is not regular, but significant. The number of studies involving hundreds of stars is also growing. Since 2010, eleven papers have published more than $250\left(T_{\mathrm{eff}}, \log g,[\mathrm{Fe} / \mathrm{H}]\right)$ determinations, and four papers present more than 500 stars (Bensby et al. 2014; Luck 2014; Ramírez et al. 2013; Sousa et al. 2011). These four datasets do not overlap greatly, except Ramírez et al. (2013) and Bensby et al. (2014), which have 156 stars in common. These two samples agree very well in their metallicity scales, with a median metallicity difference of $0.02 \mathrm{dex}(\mathrm{MAD}=0.05 \mathrm{dex})$.

Some stars in PASTEL are very well studied, with $\left(T_{\text {eff }}, \log g,[\mathrm{Fe} / \mathrm{H}]\right)$ determinations published by many different authors. As an illustration, we give in Table 1 the 36 stars that have at least 25 determinations available. The median parameters are provided together with the MAD and the number of determinations available for each parameter. The record is for the metal-poor subgiant HD 140283 with nearly 60 determinations of metallicity, which present a substantial scatter $(\mathrm{MAD}=$ $0.13 \mathrm{dex})$. The largest scatter (MAD $=0.16 \mathrm{dex})$ is for the coolest star in this subsample, the giant HD029139 ( $\alpha$ Tau). An excellent agreement between authors is obtained for several other stars, even if not of solar metallicity, like HD 022879, a dwarf of metallicity $[\mathrm{Fe} / \mathrm{H}]=-0.84(\mathrm{MAD}=0.02 \mathrm{dex})$. Figure 4 shows how metallicity varies from publication to publication. Visually, it is clear that in most cases the $[\mathrm{Fe} / \mathrm{H}]$ determinations span about 0.5 dex owing to some discrepant determinations corresponding mostly to older publications. The users who need to average all the $[\mathrm{Fe} / \mathrm{H}]$ determinations available for one star should use the median value to avoid these outliers.

There are 285 entries in PASTEL that lack a $T_{\text {eff }}$ determination because it was not given in the corresponding paper. However, we still found it useful to add these metallicities in the catalogue, even without the corresponding $T_{\text {eff }}$. Some of these metallicities come from the original $[\mathrm{Fe} / \mathrm{H}]$ catalogue by Cayrel de Strobel et al.

Abundances of elements other than iron would be very useful. It is beyond the scope of PASTEL to provide them, but we mention here several large compilations of abundances that can be cross-matched to PASTEL:

- the Hypatia Catalog (Hinkel et al. 2014) made of 84 literature sources for 50 elements across 3058 stars in the solar neighbourhood within $150 \mathrm{pc}$ of the Sun;

- chemical abundances of 1111 FGK stars (Adibekyan et al. 2012);

- homogeneous abundances from the literature for 743 stars (Soubiran \& Girard 2005);

- detailed elemental abundance study of $714 \mathrm{~F}$ and G dwarf stars in the solar neighbourhood (Bensby et al. 2014);

- the SAGA database of extremely metal-poor stars (Suda et al. 2008).

\subsection{Bibliographical references}

The main astronomical journals and the CDS database have been surveyed to search for relevant publications. The 2016 version of PASTEL includes papers until December 2015. For each publication introduced in PASTEL, the name of the first author and the bibcode are given.

Although PASTEL is intended to be exhaustive, it is likely that we have missed some papers. We recommend users of the catalogue to notify us of any missing references that should be included in the next versions of the catalogue.

\section{Stellar content of the catalogue}

In Febuary 2016 PASTEL included 31401 different stars (for 64082 records). Figure 5 displays the distribution of those stars in equatorial coordinates. The distribution is not perfectly homogeneous and the overdensities reflect the interest of astronomers in some peculiar fields, like the open clusters along the galactic plane, the Kepler field, or the galactic poles.

The histograms of $V$ and $K$ magnitudes are shown in Fig. 6. The $V$ magnitude is not available for 546 stars, while the $\mathrm{K}$ magnitude is not available for 224 stars. PASTEL is clearly dominated by bright stars, $\sim 86 \%$ of them brighter than $V=10$ and 


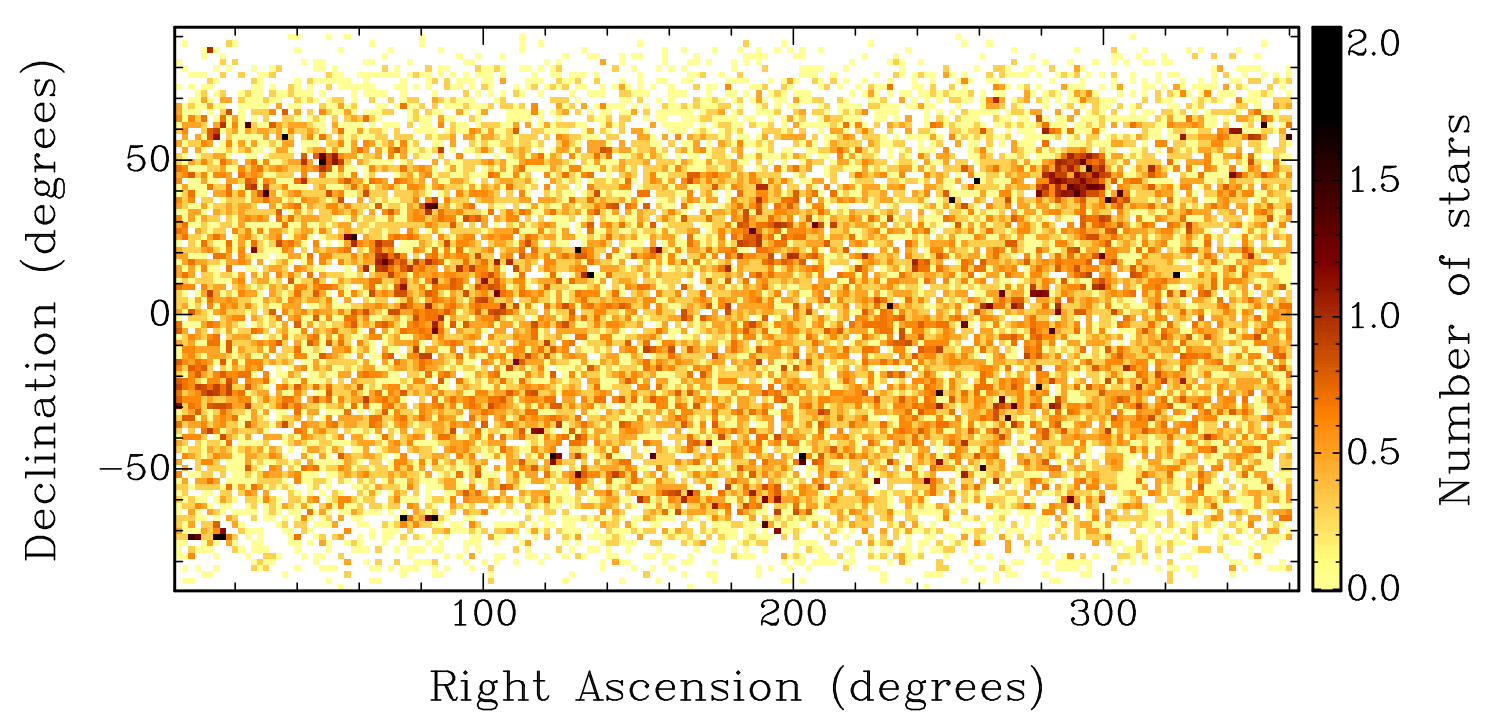

Fig. 5. Distribution of the PASTEL stars in equatorial coordinates. A logarithmic scale is used.

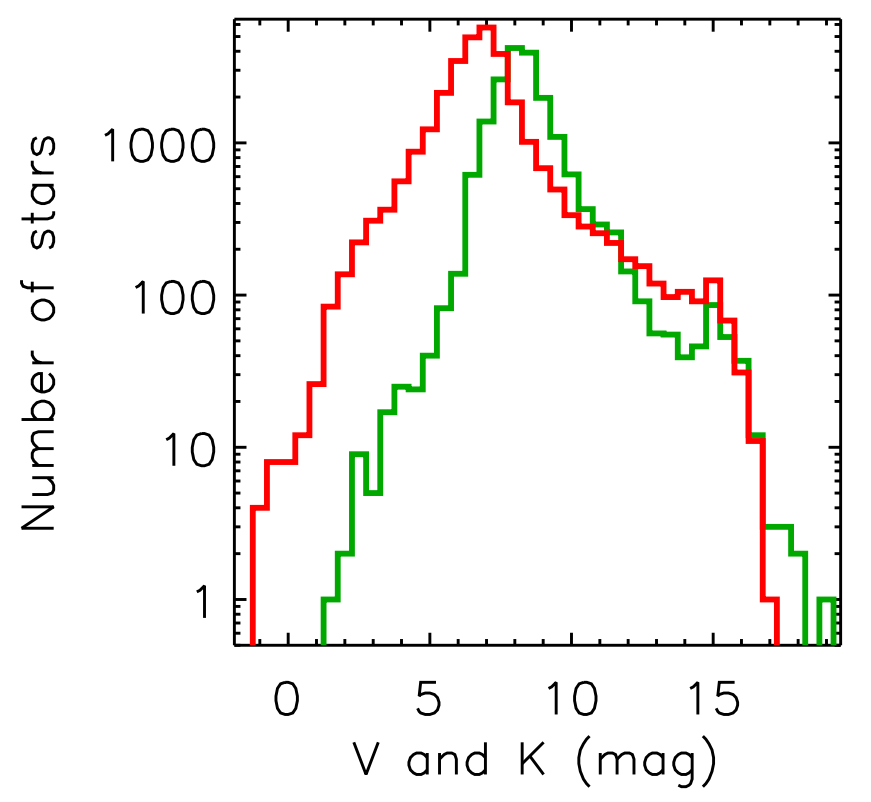

Fig. 6. Histograms of the $V$ magnitudes (green curve) and $K$ magnitudes (red curve) of the PASTEL stars.

$\sim 1 \%$ fainter than $V=15$. The fraction of faint stars has not increased since the last version of PASTEL.

There are 11197 stars in PASTEL with the full set of atmospheric parameters. This number has almost doubled since the 2010 version of the catalogue, which included 5954 such stars.

In order to show how these stars are distributed in $\left(T_{\text {eff }}, \log g,[\mathrm{Fe} / \mathrm{H}]\right)$ the median parameters have been adopted for stars with several entries. Figure 7 shows the density of the $\sim 11000$ stars in the $\log T_{\text {eff }} / \log g$ plane, while Fig. 8 shows the $T_{\text {eff }} / \log g$ diagram in four bins of metallicity corresponding roughly to the halo, the thick disc, the thin disc, and super-metal rich stars. The stellar content of the catalogue is not representative of the stellar content in the solar neighbourhood. It is biased towards stars that are massively studied in peculiar spectroscopic programmes, like solar-type stars in planet searches or metalpoor stars.

\section{Conclusion}

We have presented the new version of the PASTEL catalogue, a bibliographical compilation of $\left(T_{\text {eff }}, \log g,[\mathrm{Fe} / \mathrm{H}]\right)$ determinations. PASTEL gathers stars that have been observed at high spectral resolution and high $\mathrm{S} / \mathrm{N}$, and stars with a determination of $T_{\text {eff }}$ based on various methods. The content of the catalogue has considerably increased since its 2010 original version. The number of entries has more than doubled (30 151 determinations in 2010 vs. 64082 today), as has the number of stars with a determination of the three parameters $\left(T_{\text {eff }}, \log g,[\mathrm{Fe} / \mathrm{H}]\right)$ (5954 stars in 2010 vs. 11197 stars today).

The methods used to measure atmospheric parameters are also evolving. A novelty of PASTEL is to now include $T_{\text {eff }}$ determined from the fundamental method, with angular diameters and total flux for 151 stars, as well as $\log g$ determined from asteroseismology. The values of $\left(T_{\text {eff }}, \log g,[\mathrm{Fe} / \mathrm{H}]\right)$ are also more often determined with automated methods applied to large samples involving hundreds of stars, which is why the content of PASTEL has increased so much.

We have investigated how the errors of the parameters quoted in the literature compare with the dispersion observed from publication to publication. The agreement is good in general, showing that the errors in the literature are essentially realistic. But there are also stars that exhibit significant disagreement between spectroscopic analyses. This should lead the users of the catalogue to carefully analyse the references of the determinations they work with. The most reliable stars are those that have been studied by several different authors who agree on the parameters. In general the determinations obtained before $\sim 1990$ are less reliable because the spectra were registered on less efficient detectors, but we still found it useful to keep them in PASTEL. It is recommended that the median of the parameters be adopted instead of the mean if a single value for a given star is needed.

PASTEL offers a unique database for mining stars with known atmospheric parameters, and especially stars with a highquality spectroscopic metallicity.

Acknowledgements. We thank Alex Lobel who kindly notified us of a large number of references for hot stars. We made extensive use of the CDS Simbad and 


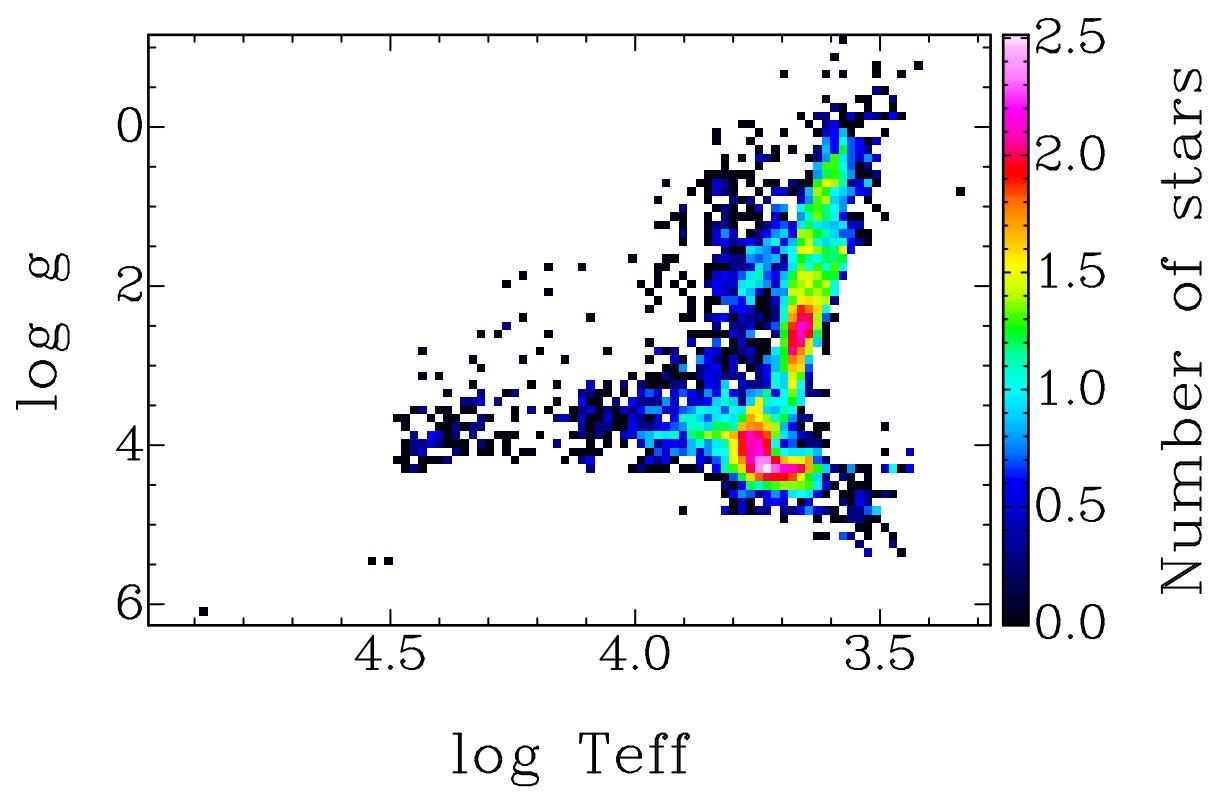

Fig. 7. Density plot of $\log \left(T_{\text {eff }}\right) / \log g$ of the PASTEL stars. A logarithmic scale is used.
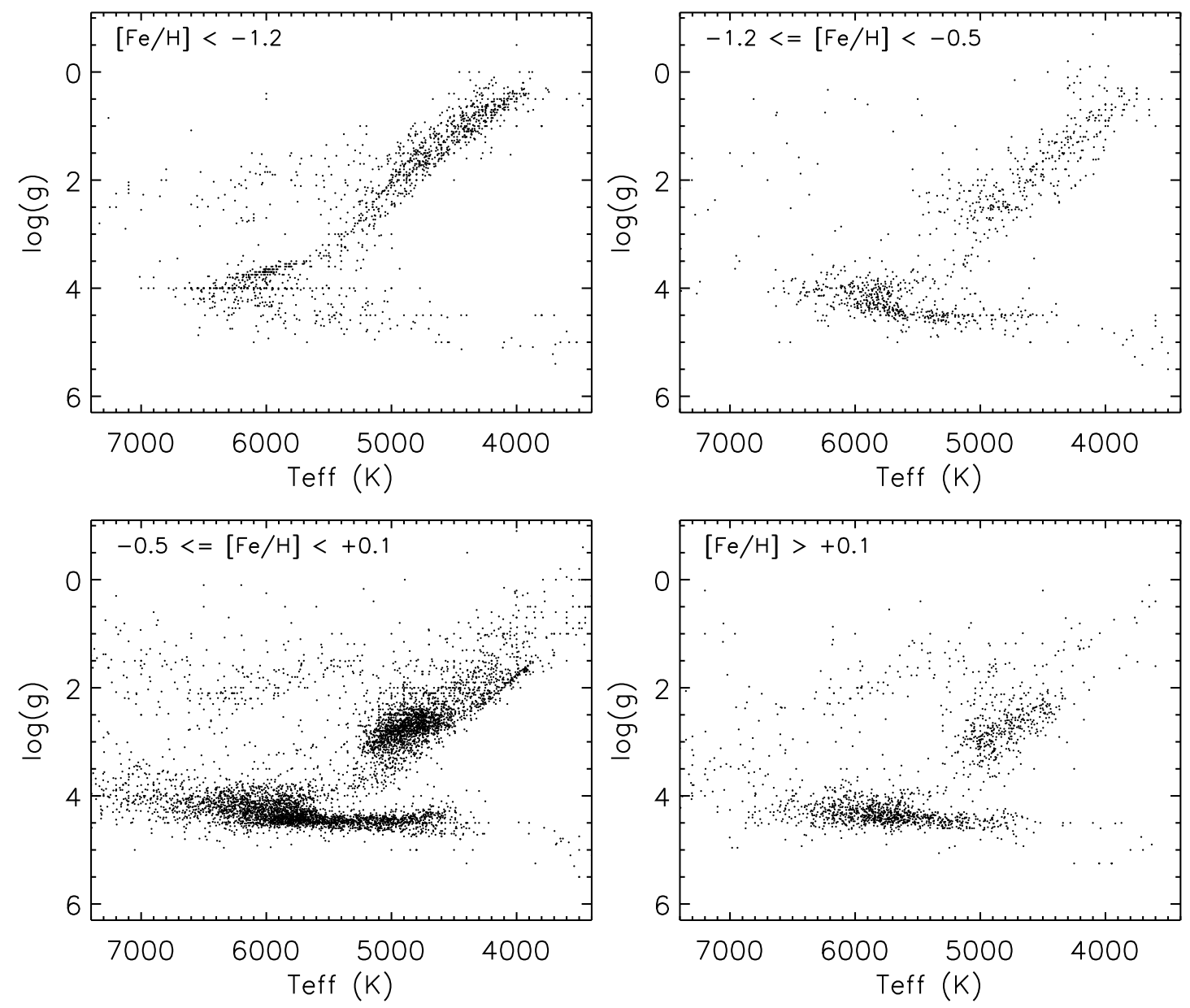

Fig. 8. $T_{\text {eff }} / \log g$ diagram of the FGK stars in several regimes of metallicity corresponding roughly to the halo (top left), to the thick disc (top right), to the thin disc (bottom left), and to metal-rich stars (bottom right). For stars with several entries in PASTEL, the median parameters have been adopted. 
VizieR services and of the NASA-ADS database, and we are extremely grateful to the staff of these services for maintaining such valuable resources.

\section{References}

Adibekyan, V. Z., Sousa, S. G., Santos, N. C., et al. 2012, A\&A, 545, A32 Aoki, W., Beers, T. C., Lee, Y. S., et al. 2013, AJ, 145, 13

Bensby, T., Feltzing, S., \& Oey, M. S. 2014, A\&A, 562, A71

Boyajian, T. S., McAlister, H. A., van Belle, G., et al. 2012a, ApJ, 746, 101 Boyajian, T. S., von Braun, K., van Belle, G., et al. 2012b, ApJ, 757, 112 Boyajian, T. S., von Braun, K., van Belle, G., et al. 2013, ApJ, 771, 40

Boyajian, T., von Braun, K., Feiden, G. A., et al. 2015, MNRAS, 447, 846 Cayrel de Strobel, G., Bentolila, C., Hauck, B., \& Curchod, A. 1980, A\&AS, 41, 405

Cayrel de Strobel, G., Bentolila, C., Hauck, B., \& Lovy, D. 1981, A\&AS, 45, 97 Cayrel de Strobel, G., Bentolila, C., Hauck, B., \& Duquennoy, A. 1985, A\&AS, 59,145

Cayrel de Strobel, G., Hauck, B., Francois, P., et al. 1992, A\&AS, 95, 273 Cayrel de Strobel, G., Soubiran, C., Friel, E. D., Ralite, N., \& Francois, P. 1997, A\&AS, 124, 299

Cayrel de Strobel, G., Soubiran, C., \& Ralite, N. 2001, A\&A, 373, 159

Creevey, O. L., Doğan, G., Frasca, A., et al. 2012, A\&A, 537, A111
Cutri, R. M., Skrutskie, M. F., van Dyk, S., et al. 2003, 2MASS All Sky Catalog of point sources, VizieR Online Data Catalog: II/246

González Hernández, J. I., \& Bonifacio, P. 2009, A\&A, 497, 497

Heiter, U., Jofré, P., Gustafsson, B., et al. 2015, A\&A, 582, A49

Hinkel, N. R., Timmes, F. X., Young, P. A., Pagano, M. D., \& Turnbull, M. C. 2014, AJ, 148, 54

Jofré, P., Heiter, U., Soubiran, C., et al. 2014, A\&A, 564, A133

Jofré, P., Heiter, U., Soubiran, C., et al. 2015, A\&A, 582, A81

Kovtyukh, V. V., Soubiran, C., Bienaymé, O., Mishenina, T. V., \& Belik, S. I. 2006, MNRAS, 371, 879

Latour, M., Fontaine, G., Chayer, P., \& Brassard, P. 2013, ApJ, 773, 84

Luck, R. E. 2014, AJ, 147, 137

Ramírez, I., Allende Prieto, C., \& Lambert, D. L. 2013, ApJ, 764, 78

Ramírez, I., Meléndez, J., Bean, J., et al. 2014, A\&A, 572, A48

Sokolov, N. A. 1995, A\&AS, 110, 553

Soubiran, C., \& Girard, P. 2005, A\&A, 438, 139

Soubiran, C., Le Campion, J.-F., Cayrel de Strobel, G., \& Caillo, A. 2010, A\&A, 515, A111

Sousa, S. G., Santos, N. C., Israelian, G., Mayor, M., \& Udry, S. 2011, A\&A, 533, A141

Suda, T., Katsuta, Y., Yamada, S., et al. 2008, PASJ, 60, 1159

Takeda, Y., \& Tajitsu, A. 2015, MNRAS, 450, 397

von Braun, K., Boyajian, T. S., van Belle, G. T., et al. 2014, MNRAS, 438, 2413 